Código JEL: M4, C4

\title{
Factores determinantes para predecir la crisis financiera en empresas argentinas
}

\author{
Norma Patricia CARO \\ Facultad de Ciencias Económicas - Universidad Nacional de Córdoba \\ pacaro@eco.unc.edu.ar \\ Margarita DIAZ \\ Facultad de Ciencias Económicas - Universidad Nacional de Córdoba \\ mdiazlujan@gmail.com
}

Recibido: 17-07-2015

Aceptado: 10-11-2015

\begin{abstract}
RESUMEN
Desde mediados del siglo veinte se ha puesto especial interés en el fracaso empresarial, que puede ser detectado a través del comportamiento de las empresas, cuyas manifestaciones se reflejan en su información contable. Los estudios preliminares utilizan metodología de corte transversal, que no incorpora la característica longitudinal de los datos. Debido a la disponibilidad de los balances de varios períodos para cada empresa y el desarrollo de métodos estadísticos avanzados para datos longitudinales, en la década del 2000 se aplicaron los denominados modelos mixtos para predecir la crisis financiera. Con la finalidad de prevenir situaciones desfavorables, tomando decisiones adecuadas, en este trabajo se evalúa el riesgo de crisis financiera de las empresas en Argentina, en el período 2003 - 2010 y se compara con el modelo obtenido por Caro, et al, 2013 para la década de 1990, comparando ambos escenarios de estabilidad económica. De esta manera se contribuye a identificar los factores determinantes de la situación de crisis de las empresas que cotizan en la Bolsa de Valores de Buenos Aires. Los ratios que miden rentabilidad y la posición de efectivo explican la mayor proporción de la heterogeneidad inducida por la
\end{abstract}


correlación que presentan los datos, lo que justifica su inclusión como coeficientes aleatorios. Los indicadores con mayor capacidad predictiva de la crisis financiera de la empresa son el índice de rentabilidad, el flujo de fondos operativos, el volumen de negocios y el índice de endeudamiento. Las tasas de clasificación correcta son mayores cuando se aplican modelos para datos longitudinales respecto a los modelos de corte transversal.

Palabras clave: Ratios Financieros, Crisis Financiera, Modelos Mixtos. Economías Emergentes.

\title{
Determinants factors in predicting the financial crisis in argentine companies
}

\begin{abstract}
Since the mid-twentieth century has been special emphasis on business failure, which can be detected through the behavior of companies, whose manifestations are reflected in their accounting information. Preliminary studies using cross-sectional methodology, it does not incorporate the longitudinal side of the data. Due to the availability of various periods balances for each company and the development of advanced statistical methods for longitudinal data, in the 2000 s the so-called mixed models to predict the financial crisis were applied. In order to prevent negative situations, taking appropriate decisions, in this study the risk of financial crisis in companies in Argentina in two scenarios of economic stability (1993 - 2000 and 2003-2010) is evaluated. In this way it helps to identify the determinants of the crisis of the companies listed on Stock Exchange of Buenos Aires. The ratios that measure profitability and cash position explains the higher proportion of heterogeneity induced by the correlated data, which justifies its inclusion as random coefficients. The index of profitability, operating cash flow, turnover and leverage ratio are the indicators most predictive ability of the financial crisis. Correct classification rates are higher when models are applied to longitudinal data instead the cross-sectional models.
\end{abstract}

Keywords: Financial Ratios, Financial crisis, Mixed Models, Emerging Economies.

Sumario: 1. Introducción 2. Revisión de la literatura 3. Método 4. Resultados 6. Conclusiones

\section{INTRODUCCIÓN}

Desde mediados del siglo veinte se ha puesto especial interés en el fracaso empresarial, que puede ser detectado a través del comportamiento de las empresas, cuyas manifestaciones se reflejan en su información contable. En ese sentido, los primeros trabajos (Altman, 1968 y Beaver, 1968) han concluido que la información contable es de utilidad para anticiparse a estos procesos de gestación e instalación 
de estados de vulnerabilidad financiera. El acceso a dicha información y el uso de herramientas estadísticas cada vez más avanzadas han contribuido al análisis de esta problemática.

Las entidades financieras se focalizan en las calificaciones crediticias y las grandes agencias de calificación de crédito han estado en observación, ya que no han tenido una predicción adecuada ante la crisis estadounidense del año 2008. Estos acontecimientos han motivado la investigación sobre nuevas formas de predecir la crisis y de realizar scoring crediticio, con técnicas econométricas avanzadas.

Con la finalidad de prevenir situaciones desfavorables, tomando decisiones adecuadas, en este trabajo se evalúa el riesgo de crisis financiera de las empresas en Argentina. De hecho, en Argentina, los estudios preliminares que se realizaron (Sandin y Porporato, 2007; Díaz, et al., 2001) desafiaron los modelos internacionales que habían sido aplicados en diferentes países (Altman, 1993), ya que con modelos propios aplicados a la economía argentina se lograron mejores evaluaciones de riesgo de empresas. No obstante ello, la metodología utilizada no incorpora la característica longitudinal de los datos.

Debido a la disponibilidad de los balances de varios períodos para cada empresa, en la década del 2000 se aplicaron los denominados modelos mixtos para predecir la crisis financiera, en economías desarrolladas (Jones y Hensher, 2004). Caro, et al (2013) estiman un modelo de riesgo de una economía emergente, como es Argentina, para la década de 1990. En este trabajo se extiende ese estudio, estimando el modelo en la siguiente década (2003 -2010) comparando los modelos obtenidos en ambos escenarios de estabilidad económica.

En los estudios mencionados en el párrafo anterior, se concluye que los modelos mixtos poseen mejor performance que el modelo logístico estándar, desde la perspectiva de las tasas de clasificación correcta. Adicionalmente, el presente trabajo contribuye a identificar los factores determinantes de la situación de crisis de las empresas que cotizan en Bolsa en el Mercado Argentino para los periodos mencionados $\mathrm{y}$, en función de ésto, predecir una posible situación financiera desfavorable ante un determinado comportamiento de esos factores.

La estructura del presente artículo consta de una breve introducción y revisión de la literatura considerando los antecedentes más relevantes. Luego se describe la metodología utilizada y los resultados obtenidos y por último se presentan las conclusiones.

\section{REVISIÓN DE LA LITERATURA}

El problema de investigación que se aborda en este trabajo está contextualizado en la teoría de la utilidad de la información contable para la toma de decisiones. El paradigma propuesto, que se denominó la "revolución contable", (Beaver, 1981), propone un cambio en la perspectiva de la medición de la ganancia económica a un enfoque "informativo". Entre los principales aspectos planteados por Beaver 
(1981), pueden mencionarse que la contabilidad ha sufrido cambios importantes, desde la década del sesenta, ya que se experimentó un incremento en los requerimientos financieros de las empresas por parte de los organismos reguladores, lo que aumenta la cantidad de datos financieros que los usuarios necesitan, y que se exponen en los estados contables. El propósito de los estados financieros es, ahora, proveer información a los dueños del capital para predecir el beneficio (futuros flujos de fondos) y analizar la eficiencia y eficacia de la actuación gerencial.

La estrecha relación existente entre los indicadores económicos-financieros construidos a partir de los estados contables de las empresas y su situación futura, justifican la construcción de modelos de pronóstico de riesgo de crisis financiera, lo que viene sucediendo desde la década de 1960 (Altman, 1968), donde la metodología seguida se basa en un diseño apareado de muestras no aleatorias y la aplicación de métodos de discriminación lineal y cuadrática. En la década de 1980 aparecen los primeros cuestionamientos al diseño no aleatorio (Olshon, 1980; Zmijewski, 1984), acompañado de la modelación con regresión logística o modelo probit (Olshon, 1980; Jones, 1987; Maddala, 1991).

Dentro de las críticas a los modelos de Altman (1968 y siguientes) se menciona el hecho de que no es conveniente su aplicación en economías emergentes sin la correspondiente adecuación, ya que los mismos son modelos construidos con información de los estados contables de países desarrollados. Por otro lado, se utiliza metodología de corte transversal, que implica el cumplimiento de supuestos que no se dan cuando se trata de datos longitudinales. Se ha probado que las tasas de error son altas (Sandín y Porporato, 2007) y que lo adecuado es la construcción de modelos con los datos propios de las empresas domésticas de cada país, considerando la información disponible.

Una revisión de la literatura más detallada sobre predicción de quiebra en general pero con especial énfasis en Latinoamérica y Argentina en particular fue presentada por Caro (2014) y se refiere a los principales trabajos de investigación realizados, tanto con métodos de corte transversal como con modelos para datos longitudinales, entre los que se destacan tanto los modelos mixtos utilizados en el presente como los modelos de duración.

El modelo logístico mixto que fuera introducido en esta temática, por Jones y Hensher (2004) es la metodología aplicada en este trabajo, considerando los dos períodos ya mencionados.

\section{MÉTODO}

a) Muestra

La población objeto de análisis está conformada por las empresas que cotizan en la Bolsa de Valores de Buenos Aires en dos períodos de tiempo.

Se pretende analizar y comparar modelos basados en datos longitudinales en dos décadas de Argentina: la del 90, caracterizada por la ley de convertibilidad y la 
estabilidad económica y la del 2000, posterior a la crisis económica del año 2001. Ambos momentos corresponden a escenarios temporales de estabilidad económica con características propias. En el primer período (1993 - 2000) Argentina tuvo niveles muy bajos de inflación en todo el periodo en que estuvo en vigencia la ley de Convertibilidad, aproximadamente diez años, algo poco frecuente en la historia económica del país y en el segundo $(2003$ - 2010) la recuperación argentina plantea un crecimiento acelerado que se debe a un cambio en el régimen cambiario que implicó una depreciación del tipo de cambio real transformando la estructura de precios relativos. Esta nueva situación creó condiciones positivas para un crecimiento liderado por las exportaciones y otorgar una protección implícita de la industria, lo que estimula la sustitución de importaciones.

No se consideraron los períodos de crisis como fueron el inmediato anterior a 1993 y el comprendido entre el 2001 y 2002. El período anterior a 1993, se caracterizó por una economía desestabilizada, luego de una grave crisis inflacionaria. El período entre el año 2000 y 2002 tuvo como desenlace la crisis de diciembre de 2001, que fue una crisis financiera generada por la restricción a la extracción de dinero en efectivo de plazos fijos, cuentas corrientes y cajas de ahorro denominada Corralito ${ }^{1}$ El gran conflicto social, la inestabilidad económica y política, afectó el comportamiento de las empresas, donde cada una debió actuar para hacer frente a la coyuntura. El hecho de considerar solo períodos de estabilidad y crecimiento se debe a que es más conveniente aplicar estos modelos donde se han tomado decisiones de inversión en función de dicha estabilidad económica. No se han tenido en cuenta periodos de inestabilidad económica, ya que pueden distorsionar el verdadero efecto que se quiere analizar (Sandin y Porporato, 2007).

El diagnóstico empresarial constituye el nexo entre el estudio - investigación y la programación de actividades mediante la detección de anomalías, para generar en la organización una situación que escape a la crisis.

En este trabajo, en primer lugar se seleccionaron las empresas que cotizan en la Bolsa de Buenos Aires y que están en crisis. Se considera que una empresa está en crisis cuando se transfieren la cotización de sus acciones a rueda reducida, esto sucede cuando la empresa solicita su concurso preventivo o bien cuando obtiene pérdidas importantes que insuman las reservas y el cincuenta por ciento $(50 \%)$ del capital ajustado o el setenta y cinco por ciento (75\%) del patrimonio neto, según lo estable el Reglamento de la Bolsa de Valores de Buenos Aires. En cuanto a las figuras del concurso preventivo y de la quiebra, ambas parten de un estado de cesación de pagos, lo que para una empresa constituye una causa inobjetable de crisis financiera.

\footnotetext{
${ }^{1}$ Consultado en Argentina Country Report publicado por The PRS Group, Inc. NY, USA, publicado en Junio de 2010 .
} 
Si bien en un grado menor al concurso preventivo o a la quiebra, no cabe duda que la transferencia a ronda reducida constituye un signo ostensible de vulnerabilidad financiera, razón por la cual en el presente trabajo se califica como empresa en crisis aquella que durante el período de análisis cotizó sus acciones por primera vez en rueda reducida.

La crisis empresaria es un estado caracterizado por estar afectada la condición de viabilidad, en la cual se produce un desequilibrio generalizado cuya principal manifestación es la disminución de la capacidad de regenerar ciclos de actividad. Se afecta a la estructura de rentabilidad y a la estructura de financiación.

De acuerdo al concepto enunciado, se seleccionaron todas las empresas en crisis en el periodo de análisis. Luego, en segundo lugar, se extrajo una muestra de empresas sanas que son aquellas firmas que no manifiestan estos signos de vulnerabilidad. La proporción de empresas sanas respecto a las empresas con problemas financieros, fue aproximadamente dos a uno en el primer período, que es el mínimo aconsejado en la literatura (Hosmer y Lemeshow, 2002), valor que se incrementa a tres a uno en el segundo.

En cuanto a los periodos analizados, la disponibilidad de la información permitió extender el rango de análisis a mayor cantidad de años en el segundo período.

Las muestras están constituidas de las siguientes maneras:

Década del 90:

Aproximadamente, 120 empresas cotizaban, en promedio, en la Bolsa de Valores de Buenos Aires en esta década, excluyendo bancos, compañías financieras y de seguro (Altman, 1968). En primer lugar se identificaron 17 empresas en crisis con información disponible y se seleccionaron 30 empresas sanas (Anexo, Tabla A1). Para cada una de las empresas en crisis se tomaron los estados contables anuales de dos a cuatro años anteriores al hecho que manifiesta el estado de crisis y para las empresas sanas se tomaron entre dos y cuatro períodos según la disponibilidad de información en esa década, lo que totaliza 150 observaciones (estados contables anuales) según lo detallado en las tablas 1 y 2 .

Tabla 1

Empresas que constituyen la muestra en la década del 90

\begin{tabular}{|c|c|c|}
\hline Estado & Observaciones & Empresas \\
\hline Sanas & 96 & 30 \\
\hline En crisis & 54 & 17 \\
\hline Total & 150 & 47 \\
\hline
\end{tabular}


Tabla 2

Periodos considerados para cada empresa, según su estado en la década del 90

\begin{tabular}{|c|c|c|}
\hline $\begin{array}{c}\text { Períodos } \\
\text { anteriores }\end{array}$ & Empresas Sanas & Empresas en crisis \\
\hline 2 & 7 & 4 \\
\hline 3 & 10 & 6 \\
\hline 4 & 13 & 7 \\
\hline Total & 30 & 17 \\
\hline
\end{tabular}

Década del 2000:

En esta década cotizan en la Bolsa de Valores de Buenos Aires, aproximadamente, 80 empresas, en promedio, sin considerar bancos, compañías financieras y de seguros. Se seleccionaron todas las empresas en crisis en el periodo, que fueron 13 y una muestra de 31 empresas sanas (Anexo, Tabla A2). Para cada una de las empresas en crisis se tomaron como mínimo dos años previos a la manifestación del estado de crisis y hasta seis periodos, según la disponibilidad de la información, lo que totaliza 238 observaciones (estados contables anuales) según lo detallado en los Tablas 3 y 4.

Tabla 3

Empresas que constituyen la muestra en la década del 2000

\begin{tabular}{|c|c|c|}
\hline Estado & Observaciones & Empresas \\
\hline Sanas & 186 & 31 \\
\hline En crisis & 52 & 13 \\
\hline Total & 238 & 44 \\
\hline
\end{tabular}

Tabla 4

Periodos considerados para cada empresa, según su estado en la década del 2000

\begin{tabular}{|c|c|c|}
\hline $\begin{array}{c}\text { Períodos } \\
\text { anteriores }\end{array}$ & Empresas Sanas & Empresas en crisis \\
\hline Hasta 4 & - & 6 \\
\hline Mas de 4 & 31 & 7 \\
\hline Total & 31 & 13 \\
\hline
\end{tabular}

b) Variables

La variable respuesta es de característica dicotómica: empresas en crisis y sanas, tal como se definieron en la sección anterior.

En cuanto a las variables independientes que se utilizan en este estudio, se definen diversos ratios financieros, tamaño de la empresa y sector de la economía al que pertenece. 
Los ratios financieros se calculan a través de la información disponible en los estados contables de las empresas cotizantes. Las razones o ratios sobre los cuales se realiza el análisis e interpretación de los estados financieros nos permite, no sólo resolver algunos aspectos relacionados a la toma de decisiones, sino cuantificar la probabilidad de pertenecer al grupo de empresas sanas o en crisis.

Tradicionalmente los ratios representaban datos para el análisis descriptivo, mientras que a partir de la década de 1960 son utilizados como una herramienta predictiva (Beaver, 1966, 1968 y Altman, 1968). De allí el uso de los ratios financieros para medir características de la empresa que la llevarán a evitar el fracaso empresarial, debido a que se pueden detectar signos de deterioro en el comportamiento de los mismos. Una de las debilidades en el uso de los ratios, tiene que ver con la desproporcionada cantidad de ellos que se utilizan en las investigaciones y como no existe una teoría solida sobre que ratios deben utilizarse, la literatura ha proporcionado una respuesta al respecto.

En la década de 1980 comienzan los estudios que incluyen el cash flow operativo a fin de investigar si este indicador posee mayor capacidad predictiva que otros ratios individuales, para lo cual se lo aplicó conjuntamente. El cash flow total incluye componentes operativos, de inversión y financiación. El operativo fue el más usado, con diferentes criterios. La nueva tendencia metodológica indica que debe incluirse este ratio junto con otros que se obtienen por el método de lo devengado para la predicción de la crisis empresarial.

Los ratios que se consideran en este trabajo son indicadores calculados con la información de los estados contables presentados (Altman, 1968 y Jones y Hensher, 2004).

- Ratio de Flujo de Fondos (FF_AT): mide la posición de efectivo originado por las actividades operativas respecto al total del activo.

$$
\text { FF_AT }=(\text { FFO/AT }) * 100
$$

FFO: flujo de fondos generado por las operaciones AT: Activo Total

- Ratio de Liquidez (E_AT): mide la proporción de los recursos más líquidos de la empresa (disponibilidades e inversiones que pueden hacerse efectivas dentro de las 24 horas) sobre el total de activo.

$$
E_{-} A T=(E / A T) * 100
$$

E: disponibilidades (efectivo e inversiones que se convierten en efectivo dentro de las $24 \mathrm{hs}$.)

AT: Activo Total 
- Ratio de Rotación del Activo Total (V_AT): es el coeficiente que mide la relación entre las ventas y el activo total.

$$
\text { V_AT }=(\mathbf{V} / A T) * 100
$$

V: total de ventas

AT: Activo Total

- Ratio de Endeudamiento (D/PN): mide la relación de las deudas (capital de terceros) sobre el capital aportado por los dueños de la empresa.

$$
\text { D_PN }=(\mathbf{D} / \mathbf{P N}) * 100
$$

D: total de deudas

PN: Patrimonio Neto

- Ratio de Capital de trabajo (CT_AT): expresa el grado de fluidez de los activos e indica en qué medida la inmovilización del activo total resulta neutralizada por el capital de trabajo.

$$
\text { CT_AT }=(C T / A T) * 100
$$

CT: Capital de Trabajo (Activo Corriente menos Pasivo Corriente) AT: Activo Total

- Ratio de rentabilidad económica (GE_AT): mide la rentabilidad en función de las ganancias de la explotación, la capacidad básica de generación de utilidades.

$$
\text { GE_AT }=(\text { UaiI } / A T) * 100
$$

UaiI: Utilidad antes de intereses e impuestos AT: Activo Total

En cuanto a la variable tamaño, se midió como el logaritmo natural del activo total. Esta variable ha sido agregada por Altman, et al. (1977) a fin de considerar el efecto que tiene el tamaño de la empresa para la capacidad predictiva del modelo.

\section{c) Modelo}

En un comienzo se aplicaron modelos de clasificación supervisada para datos de corte transversal, considerando el análisis un año antes de la manifestación de crisis, o bien dos años antes o tres, según las diferentes investigaciones (entre 1960 y 2000).

En los métodos de corte transversal, se parte de una muestra de elementos con una cierta estructura de grupo, con el objetivo de determinar una regla de 
clasificación que permita evaluar las agrupaciones de dichas observaciones y clasificar elementos futuros, en base a dicha muestra.

Cada objeto o elemento de análisis se asigna a un grupo en función de la información que se conoce de él. Se desea que dicha asignación sea de manera tal que minimice alguna medida de error.

En la actualidad (desde el año 2000 en adelante) los modelos que consideran la historia de las empresas, es decir varios ejercicios anuales, son los convenientes para explicar y predecir la crisis financiera. Por ello, se aplican lo modelos mixtos (Jones y Hensher, 2004) en cada periodo de tiempo.

Con ambos métodos, se obtuvieron altas tasas de error tipo I (Tabla 5), siendo este error el más grave debido a que se clasifica una empresa en crisis como si fuera sana, lo que estaría ocultando la verdadera realidad y podría traer consecuencias graves, mientras que el error tipo II, que mide la clasificación de una empresa sana como que no lo está, no sería tan negativo, a lo sumo se tomarían medidas preventivas que se podrían suponer que no perjudicarían a la empresa. Con ambas metodologías la tasa de clasificación correcta fue del $74 \%$ para el primer modelo y del 75,3\% para el segundo.

\section{Tabla 5}

Tasas de clasificaron correcta y tasas de error con métodos de corte transversal (Década del 90)

\begin{tabular}{|l|c|c|}
\hline \multicolumn{1}{|c|}{ Porcentajes } & $\begin{array}{c}\text { Análisis } \\
\text { Discriminante } \\
\text { Lineal }\end{array}$ & $\begin{array}{c}\text { Regresión } \\
\text { Logística }\end{array}$ \\
\hline Error Tipo I & 59,3 & 61,1 \\
\hline Error tipo II & 7,3 & 4,2 \\
\hline \% de clasificación correcta & 74 & 75,3 \\
\hline
\end{tabular}

\section{Modelos Logístico Mixto}

En la década de 1980 fue propuesta una clase general de modelos para datos longitudinales que pretenden resolver los problemas comentados, incluyendo estructuras para la covarianza, como son los modelos lineales mixtos. La forma general de un Modelo Lineal Mixto está expresada en (1):

$$
Y_{i j}=\mathbf{X}_{i j}^{\prime} \boldsymbol{\beta}+\mathbf{Z}_{i j}^{\prime} \mathbf{b}_{i}+e_{i j}
$$

Donde $\mathbf{Z}_{i j}$ es un vector de diseño para los efectos aleatorios, $\mathbf{b}_{j} \sim N(\mathbf{0}, G)$ y el término de error, $e_{i j} \sim N\left(\mathbf{0}, V_{i}\right)$. Comúnmente, se asume que la matriz de covarianzas del término del error es $V_{j}=\sigma^{2} I$. 
Cuando la variable respuesta es discreta, los modelos lineales no son apropiados para medir los cambios en la respuesta según las covariables, por lo que se han desarrollado los Modelos Lineales Generalizados para datos longitudinales. La característica principal de estos modelos es que se asume una adecuada transformación no lineal de la media de la respuesta para trabajar con una función lineal de las covariables.

En esta oportunidad se aplica un modelo para datos longitudinales donde la variable respuesta es la variable binaria que indica el grupo al que pertenece la empresa y las variables predictoras son indicadores económicofinancieros, tamaño y sector.

En primer lugar se aplicó un modelo con un solo efecto aleatorio, (Caro et al., 2013) el índice de flujo de fondos operativos respecto del activo total, cuya inclusión se debe a que el mismo explica en mayor proporción la heterogeneidad inducida por la correlación de los datos. La formulación del modelo es:

$$
\begin{gathered}
\operatorname{logit}\left(\pi_{i j}\right)=\beta_{0}+\left(\beta_{1}+b_{1 j}\right) F F_{-} A T_{i j}+\beta_{2} G E_{-} A T_{i j}+\beta_{3} E_{-} A T_{i j}+\beta_{4} V_{-} A T_{i j}+ \\
+\beta_{5} D_{-} P N_{i j}+\tilde{\beta_{6}} C T_{-} A T_{i j} \\
\mathrm{~b}_{j} / \mathbf{x}_{i j} \quad N\left(0, d_{11}\right) \\
\mathrm{i}: 1, \ldots \mathrm{n} \quad \mathrm{j}: 1, \ldots, \mathrm{n}_{i}
\end{gathered}
$$

$n$ : representa la cantidad de empresas y $n_{i}$ la cantidad de períodos anuales.

$\beta_{k}$ : son los coeficientes de cada una de las $k$ covariables.

$\mathrm{b}_{j}$ : efecto aleatorio para cada empresa.

$d_{11}$ : varianza del efecto aleatorio.

En este trabajo, se incorpora un nuevo modelo con dos coeficientes aleatorios independientes en el predictor lineal (Modelo 2), el índice de rentabilidad (GE_AT) y el índice de flujo de fondos operativos (FF_AT), tiene la siguiente formulación:

Donde

$$
\begin{aligned}
\operatorname{logit}\left(\pi_{i j}\right)= & \beta_{0}+\left(\beta_{1}+b_{1 j}\right) G E_{-} A T_{i j}+\left(\beta_{2}+b_{2 j}\right) F F_{-} A T_{i j}+ \\
& \beta_{3} E_{-} A T_{i j}+\beta_{4} V_{-} A T_{i j}+\beta_{5} D_{-} P N_{i j}+\beta_{6} C T_{-} A T_{i j}
\end{aligned}
$$

$$
\pi_{i j}=P\left(y_{i j}=1 / \mathbf{x}_{i j}, \mathbf{b}_{j}\right)
$$




$$
\mathbf{b}_{j}=\left[\begin{array}{l}
b_{1 j} \\
b_{2 j}
\end{array}\right] / \mathbf{x}_{i j} \sim \quad N_{2}(0, \Psi) \quad \Psi=\left(\begin{array}{cc}
d_{11} & 0 \\
0 & d_{22}
\end{array}\right)
$$

$\Psi$ : matriz de varianzas de los efectos aleatorios (independientes).

\section{RESULTADOS}

Comparando ambos modelos y contrastando con pruebas de razón de verosimilitud, se concluye que el modelo que incorpora dos efectos aleatorios es superior al modelo con un solo efecto aleatorio (Tabla 6). De manera que los ratios que miden la rentabilidad (GE_AT) y el flujo de fondos operativo de la empresa (FF_AT) resultan adecuados para explicar la mayor proporción de la heterogeneidad inducida por los datos, lo que justifica su inclusión como coeficientes aleatorios en el predictor lineal, los cuales se suponen independientes y con distribución normal.

El modelo fue ajustado por máxima verosimilitud, con la rutina NLMIXED del software SAS. La significatividad de la varianza del efecto aleatorio se obtuvo con la prueba de razón de verosimilitud (LRT), contrastando contra un modelo sin efectos aleatorios.

\section{Tabla 6}

Pruebas de razón de verosimilitud para selección del modelo con dos efectos aleatorios (Década del 90)

\begin{tabular}{|l|c|c|c|}
\hline \multicolumn{1}{|c|}{ Modelo propuesto } & $\mathbf{- 2 \operatorname { l o g } ( V e r o s i m i l i t u d )}$ & Dif (1) & p-value \\
\hline $\begin{array}{l}\text { Modelo con un efecto } \\
\text { aleatorio: FF_AT (Modelo 1) }\end{array}$ & 115,20 & \multicolumn{2}{|l|}{} \\
\hline $\begin{array}{l}\text { Modelo con dos efectos } \\
\text { aleatorios: GE_AT y FF_AT } \\
\text { (Modelo 2) }\end{array}$ & 103,80 & 11,40 & $\mathbf{0 , 0 0 0 4}$ \\
\hline
\end{tabular}

(1) Dif: diferencia entre $-2 \log ($ Verosimilitud) del modelo propuesto y del modelo con un efecto aleatorio.

La inclusión de estos coeficientes aleatorios permitió identificar aquellos indicadores con mayor capacidad predictiva de la crisis financiera de la empresa. Se advierte que la mayoría de los ratios son significativos $(\alpha=0,10)$ excepto Capital de Trabajo sobre Activo Total (CT_AT) y Efectivo sobre Activo Total (E_AT) (Tabla 7). 
Tabla 7

Estimaciones de los parámetros del modelo con dos efectos aleatorios (Década de 1990)

\begin{tabular}{|c|c|c|c|c|}
\hline Efectos Fijos & Coeficiente & $\begin{array}{c}\text { Error } \\
\text { Estándar }\end{array}$ & p-value & $\begin{array}{c}\text { Odd Ratios } \\
\text { variables } \\
\text { significativas }\end{array}$ \\
\hline Constante & 0,5393 & 1,9314 & 0,7813 & \\
\hline GE_AT & $-1,2379$ & 0,5529 & $\mathbf{0 , 0 3 0 2}$ & 0,29 \\
\hline FF_AT & $-0,2412$ & 0,1246 & $\mathbf{0 . 0 5 9 3}$ & 0.79 \\
\hline E_AT & $-0,1462$ & 0,1276 & 0.2581 & \\
\hline V_AT & $-0,0939$ & 0,0408 & $\mathbf{0 . 0 2 6 2}$ & 0,91 \\
\hline D_PN & 0,0302 & 0,0131 & $\mathbf{0 . 0 2 6 3}$ & 1,03 \\
\hline CT_AT & 0,1137 & 0,1114 & 0.3129 & \\
\hline
\end{tabular}

Ordenando los ratios, en primer lugar se posiciona el índice que mide rentabilidad (GE AT), el cual indica que por cada aumento unitario en dicho ratio, la chance de entrar en estado de crisis disminuye en un $71 \%$. En segundo lugar el índice de posición de efectivo (FF_AT) cuyo odd ratio $(0,79)$ muestra que un incremento unitario en dicho índice disminuirá esa chance en aproximadamente un $21 \%$. Siguen en importancia el índice de rotación (V_AT), que indica que por cada incremento unitario de este índice disminuirá la chance de crisis en un $9 \%$. Por último, el índice de endeudamiento (D_PN), al que le corresponde un odd ratio de 1,03 , lo que significa que por cada unidad que aumenta el ratio, la chance de ingresar a un estado de crisis se incrementa en un $3 \%$. Como se advierte la inclusión de un segundo efecto aleatorio permite captar la incidencia de los efectos fijos estimados.

Por último, a fin de validar el modelo, se calcula la tasa de error aparente. La tabla 8 muestra que el modelo con dos coeficientes aleatorios posee mejor tasa de clasificación aparente que los modelos de corte transversal (ver Tabla 5), no obteniéndose ninguna empresa mal clasificada para esta muestra.

Tabla 8

Tasas de clasificación correcta y tasas de error con Modelos Mixtos (Década del 90)

\begin{tabular}{|l|c|c|}
\hline \multicolumn{1}{|c|}{ Porcentajes } & $\begin{array}{c}\text { Modelo 1 } \\
\text { Un efecto aleatorio }\end{array}$ & $\begin{array}{c}\text { Modelo 2 } \\
\text { Dos efectos } \\
\text { aleatorios }\end{array}$ \\
\hline Error Tipo I & 9,26 & 0 \\
\hline Error tipo II & 7,29 & 0 \\
\hline \% de clasificación correcta & 91,23 & 100,0 \\
\hline
\end{tabular}




\section{Aplicación de modelos en empresas en la década del 2000}

En este periodo de tiempo se aplicaron también modelos de corte transversal y luego los modelos mixtos. De la comparación, se deduce, nuevamente, que los modelos mixtos tienen mejor desempeño que los de corte transversal. La tabla 9 muestra que las tasas de error tipo I son muy elevadas con el análisis discriminante lineal y la regresión logística.

Tabla 9

Tasas de clasificaron correcta y tasas de error con métodos de corte transversal (Década del 2000)

\begin{tabular}{|l|c|c|}
\hline \multicolumn{1}{|c|}{ Porcentajes } & $\begin{array}{c}\text { Análisis } \\
\text { Discriminante Lineal }\end{array}$ & Regresión Logística \\
\hline Error Tipo I & 42,3 & 26,9 \\
\hline Error tipo II & 1,6 & 1,6 \\
\hline \% de clasificación correcta & 89,5 & 92,9 \\
\hline
\end{tabular}

En esta década, como en la anterior, se hicieron numerosas pruebas con los distintos ratios como efectos fijos y aleatorios, seleccionándose los modelos con mejores resultados y performance. En las empresas de esta década se aplicó también un modelo con los ratios financieros ya descriptos (Modelo 3) con un efecto aleatorio, que es el índice de rentabilidad.

La estructura del modelo es la siguiente:

$$
\begin{aligned}
\operatorname{logit}\left(\pi_{i j}\right)= & \beta_{0}+\left(\beta_{1}+b_{1 j}\right) G E_{-} A T_{i j}+\beta_{2} F F_{-} A T_{i j}+\beta_{3} E_{-} A T_{i j}+\beta_{4} V_{-} A T_{i j}+ \\
(12) & +\beta_{5} D_{-} P N_{i j}+\beta_{6} C T_{-} A T_{i j}+\beta_{7} L N A_{i j}
\end{aligned}
$$

donde

$$
\begin{aligned}
& \pi_{i j}=P\left(y_{i j}=1 / \mathbf{x}_{i j}, \mathrm{~b}_{j}\right) \\
& \mathrm{b}_{j} / \tilde{\mathbf{x}}_{i j} \quad N\left(0, d_{11}\right)
\end{aligned}
$$

En este modelo el índice de rentabilidad es significativo (Tabla 10), ya que el mismo explica la mayor proporción de la variabilidad total. 
Tabla 10

Pruebas de razón de verosimilitud para selección del modelo de un coeficiente aleatorio (Década del 2000)

\begin{tabular}{|l|c|c|c|}
\hline \multicolumn{1}{|c|}{ Modelo propuesto } & $\mathbf{- 2 \operatorname { l o g } ( \text { Verosimilitud) }}$ & Dif (1) & p-value \\
\hline Modelo sin efectos aleatorios & 84,68 & \multicolumn{2}{|c|}{} \\
\hline $\begin{array}{l}\text { Modelo con un efecto } \\
\text { aleatorio: GE_AT (Modelo 3) }\end{array}$ & 77,90 & 6,78 & $\mathbf{0 , 0 0 4 6}$ \\
\hline
\end{tabular}

(1) Dif: diferencia entre $-2 \log$ (Verosimilitud) del modelo propuesto y del modelo sin efectos aleatorios

En cuanto a los efectos fijos (Tabla 10) resultan significativos a un $10 \%$, el índice de rentabilidad, el de rotación, el de endeudamiento y el tamaño de la empresa. A diferencia de los modelos construidos con las empresas de la década anterior, el tamaño, medido a través del logaritmo del activo total es significativo, es decir que mientras más pequeña es la empresa, aumenta la chance de entrar en crisis.

Respecto a los ratios, ante una disminución unitaria del índice de rentabilidad, la chance de presentar crisis aumenta en un $14 \%$. Luego le sigue el índice de rotación que indica que ante una disminución en el nivel de ventas, la chance de crisis aumenta en un $7 \%$ y por último, ante un aumento unitario en el nivel de endeudamiento, la empresa aumenta en un $2 \%$ de posibilidad de ingresar a un estado de crisis (Tabla 11).

\section{Tabla 11}

Estimaciones de los parámetros del modelo con un coeficiente aleatorio (Década del 2000)

\begin{tabular}{|c|c|c|c|c|}
\hline Efectos Fijos & Coeficiente & $\begin{array}{c}\text { Error } \\
\text { Estándar }\end{array}$ & p-value & $\begin{array}{c}\text { Odd Ratios } \\
\text { variables } \\
\text { significativas }\end{array}$ \\
\hline Constante & 30,965 & 7,951 & 0,0003 & \\
\hline GE_AT & $-0,1473$ & 0,080 & $\mathbf{0 , 0 7 3 9}$ & 0,86 \\
\hline FF_AT & $-0,0644$ & 0,0418 & 0.1306 & \\
\hline E_AT & 0,0467 & 0,2445 & 0.8494 & \\
\hline V_AT & $-0,0744$ & 0,0204 & $\mathbf{0 . 0 0 0 7}$ & 0,93 \\
\hline D_PN & 0,0157 & 0,0043 & $\mathbf{0 . 0 0 0 7}$ & 1,02 \\
\hline CT_AT & 0,0234 & 0,0144 & 0.1069 & \\
\hline LNA & $-1,551$ & 0,388 & $\mathbf{0 , 0 0 0 3}$ & 0,21 \\
\hline
\end{tabular}

Con dos coeficientes aleatorios, el tamaño de las empresas no fue significativo, por lo que, a los fines comparativos, con la década del 90, se realizó otro modelo 
mixto (Modelo 4) incluyendo como covariables solo los ratios financieros. En este caso, los dos efectos aleatorios resultaron significativos, el índice de flujo de fondos operativos y el índice de rentabilidad (Tabla 12).

$$
\begin{aligned}
\operatorname{logit}\left(\pi_{i j}\right)= & \beta_{0}+\left(\beta_{1}+b_{1 j}\right) G E_{-} A T_{i j}+\left(\beta_{2}+b_{2 j}\right) F F_{-} A T_{i j}+ \\
& \beta_{3} E_{-} A T_{i j}+\beta_{4} V_{-} A T_{i j}+\beta_{5} D_{-} P N_{i j}+\beta_{6} C T_{-} A T_{i j}
\end{aligned}
$$

Respecto a los efectos fijos, los índices que resultaron significativos fueron los mismos que se obtuvieron en los modelos construidos para la década anterior, esto es el índice de flujo de fondos operativos, de rentabilidad, de rotación y de endeudamiento. Para los tres primeros, ante cambios unitarios en cada uno de ellos, la chance de disminución de crisis es del $24 \%, 15 \%$ y $7 \%$ respectivamente. Por otro lado, a mayor nivel de endeudamiento, la chance de ingresar en crisis es del $1 \%$ (Tabla 13).

\section{Tabla 12}

Pruebas de razón de verosimilitud para selección del modelo con dos efectos aleatorios (Década del 2000)

\begin{tabular}{|l|c|c|c|}
\hline \multicolumn{1}{|c|}{ Modelo propuesto } & $\mathbf{- 2 \operatorname { l o g } ( V e r o s i m i l i t u d )}$ & Dif (1) & p-value \\
\hline $\begin{array}{l}\text { Modelo con un efecto } \\
\text { aleatorio: GE_AT }\end{array}$ & 112,7 & \multicolumn{2}{|l|}{} \\
\hline $\begin{array}{l}\text { Modelo con dos efectos } \\
\text { aleatorios: GE_AT y FF_AT } \\
\text { (Modelo 4) }\end{array}$ & 105,3 & 7,40 & $\mathbf{0 , 0 0 3 3}$ \\
\hline
\end{tabular}

(1) Dif: diferencia entre $-2 \log$ (Verosimilitud) del modelo propuesto y el modelo con un efecto aleatorio.

Tabla 13

Estimaciones de los parámetros del modelo con dos efectos aleatorios (Década del 2000)

\begin{tabular}{|c|c|c|c|c|}
\hline Efectos Fijos & Coeficiente & $\begin{array}{c}\text { Error } \\
\text { Estándar }\end{array}$ & p-value & $\begin{array}{c}\text { Odd Ratios } \\
\text { variables } \\
\text { significativas }\end{array}$ \\
\hline Constante & 1,2269 & 0,9009 & 0,1805 & \\
\hline GE_AT & $-0,1620$ & 0,0920 & $\mathbf{0 , 0 8 5 5}$ & 0,85 \\
\hline FF_AT & $-0,2692$ & 0,1212 & $\mathbf{0 . 0 3 1 8}$ & 0,76 \\
\hline E_AT & 0,3635 & 0,2328 & 0.1259 & \\
\hline V_AT & $-0,0760$ & 0,0217 & $\mathbf{0 . 0 0 1 1}$ & 0,93 \\
\hline D_PN & 0,0146 & 0,0066 & $\mathbf{0 . 0 3 0 8}$ & 1,01 \\
\hline CT_AT & 0,0077 & 0,0108 & 0.4806 & \\
\hline
\end{tabular}


Respecto a la tasa de clasificación correcta es mayor que la obtenida en los métodos de corte transversal y a su vez, es mayor en el modelo que incluye dos efectos aleatorios (Tabla 14). En ambos períodos de tiempo resultaron significativos los mismos ratios.

Tabla 14

Tasas de clasificación correcta y tasas de error con Modelos Mixtos (Década del

\begin{tabular}{|l|c|c|}
\hline \multicolumn{1}{|c|}{ Porcentajes } & $\begin{array}{c}\text { Modelo 3 } \\
\text { Un efecto aleatorio } \\
\text { Covariables: ratios y } \\
\text { tamaño }\end{array}$ & $\begin{array}{c}\text { Modelo 4 } \\
\text { Dos efectos } \\
\text { aleatorios } \\
\text { Covariables: ratios }\end{array}$ \\
\hline Error Tipo I & 17,31 & 15,38 \\
\hline Error tipo II & 0,54 & 0,54 \\
\hline $\begin{array}{l}\text { \% de clasificación } \\
\text { correcta }\end{array}$ & 95,80 & 96,22 \\
\hline
\end{tabular}

Una síntesis de los modelos construidos se muestra en la Tabla 17, donde se observa que los ratios financieros poseen poder predictivo y que los que resultaron significativos para las empresas del Mercado Argentino en los diferentes espacios temporales analizados, fueron: el índice de flujos de fondos operativos (FF_AT), el índice de rentabilidad (GE_AT), el de rotación (V_AT) y el de endeudamiento (D_PN).

Tabla 15

Resumen de Modelos Mixtos construidos

\begin{tabular}{|l|c|c|c|c|c|c|}
\hline \multirow{2}{*}{ Modelos } & \multirow{2}{*}{ Décadas } & \multicolumn{2}{|c|}{ Efectos significativos } & \multicolumn{3}{c|}{ Tasas } \\
\cline { 3 - 7 } & Aleatorios & Fijos & $\begin{array}{c}\text { Error tipo } \\
\text { I }\end{array}$ & $\begin{array}{c}\text { Error Tipo } \\
\text { II }\end{array}$ & $\begin{array}{c}\text { Clasificación } \\
\text { correcta }\end{array}$ \\
\hline Modelo 1 & 1990 & FF_AT & $\begin{array}{c}\text { GE_AT } \\
\text { V_AT } \\
\text { D_PN }\end{array}$ & 9,26 & 7,29 & 91,23 \\
\hline Modelo 2 & 1990 & $\begin{array}{l}\text { FF_AT } \\
\text { GE_AT }\end{array}$ & $\begin{array}{c}\text { FF_AT } \\
\text { GE_AT } \\
\text { V_AT } \\
\text { D_PN }\end{array}$ & $\mathbf{0 , 0}$ & $\mathbf{0 , 0}$ & $\mathbf{1 0 0 , 0}$ \\
\hline Modelo 3 & 2000 & GE_AT & $\begin{array}{c}\text { GE_AT } \\
\text { V_AT } \\
\text { D_PN } \\
\text { LNA }\end{array}$ & 17,31 & 0,54 & 95,80 \\
\hline Modelo 4 & 2000 & $\begin{array}{l}\text { FF_AT } \\
\text { GE_AT }\end{array}$ & $\begin{array}{c}\text { FF_AT } \\
\text { GE_AT } \\
\text { V_AT } \\
\text { D_PN }\end{array}$ & $\mathbf{1 5 , 3 8}$ & $\mathbf{0 , 5 4}$ & $\mathbf{9 6 , 2 2}$ \\
\hline
\end{tabular}




\section{CONCLUSIONES}

En el presente trabajo se identificaron los factores determinantes de la situación de crisis de las empresas que cotizan en Bolsa en el Mercado Argentino para el periodo 2003-2010 y se compararon con los obtenidos en la década de 1990. En función de esto, es posible predecir una posible situación financiera desfavorable ante un determinado comportamiento de esos indicadores.

Los modelos mixtos son adecuados cuando la estructura de los datos introduce dependencia en las respuestas múltiples dentro de cada unidad, lo que es un aporte en términos de modelos avanzados y resultaron más efectivos en la predicción de crisis, en economías emergentes.

Si bien las variables significativas son las mismas que se detectan en los modelos de corte transversal, la clasificación a la que se arriba con la versión mixta del modelo logístico es claramente superior a la obtenida con la regresión logística estándar. En efecto, las tasas de clasificación correcta son mayores cuando se aplican modelos para datos longitudinales respecto a los modelos de corte transversal y dentro de esos modelos, los que tienen dos efectos aleatorios respecto a los que tienen uno.

En ambas décadas, cuando se consideran como predictores sólo los ratios financieros, los que miden rentabilidad y posición de efectivo explican la mayor proporción de la heterogeneidad inducida por la correlación que presentan los datos, lo que justifica su inclusión como coeficientes aleatorios.

Los indicadores con mayor capacidad predictiva de la crisis financiera de la empresa son el índice de rentabilidad, el flujo de fondos operativos, el volumen de negocios y el índice de endeudamiento.

Una de las limitaciones del trabajo tiene que ver con la cantidad de empresas cotizantes, ya que son pocas, lo que es una constante en las economías latinoamericanas. No obstante ello, al considerar varios periodos para cada empresa, la cantidad de datos con los que trabajan los modelos es mayor.

Dentro de las futuras líneas de investigación existe la posibilidad de aplicar estos modelos en otras economías latinoamericanos a fin de comparar los factores que determinan en cada mercado, la posibilidad de crisis.

\section{REFERENCIAS}

ALTMAN, E. (1968), Financial ratios, discriminant analysis and the prediction of corporate bankruptcy, Journal of Finance, Vol. 23 (4), 589-609.

ALTMAN, E.; HALDEMAN, R. and NARAYANAN, P. (1977), ZETA analysis: a new model to identify bankruptcy risk of corporations, Journal of Banking and Finance, Vol. 1 (1), 29-54.

ALTMAN, E. (1993), Corporate Financial Distress and Bankruptcy, John Wiley and Sons, New York. 
BEAVER, W. (1966), Financial ratios as predictors of failures. Empirical research in accounting selected studies 1966, Journal of Accounting Research, Vol. 5 (supplement), 71-111.

BEAVER, W. (1968), Alternative Accounting Measures as Predictors of Failure, The Accounting Review, 113-122.

BEAVER, W. (1981), Financial reporting: an Accounting Revolution, Prentice Hall, USA.

CARO, N, DÍAZ, M y PORPORATO, M (2013), Predicción de quiebras empresariales en economías emergentes: uso de un modelo logístico mixto, Revista de Métodos Cuantitativos para la economía y la empresa, Vol. 16, 200-215.

CARO, N (2014), Modelos de predicción de crisis financiera en empresas: una revisión de la literatura, Revista Internacional Legis de Contabilidad y Auditoría, Vol. 58, 135-183.

DÍAZ, M, FERRERO, F, DÍAZ, C, STIMOLO, M y CARO, N. (2001), Perfomance del Análisis Discriminante Regularizado y la Regresión Logística en la Predicción de Crisis Financieras, Revista de la Sociedad Argentina de Estadística, Vol. 5 (1-2), 33-45.

HOSMER, DW y LEMESHOW, S (2000), Appied logistic regression, Wiley, New York.

JONES, F. (1987), Current techniques in bankruptcy prediction, Journal of Accounting Literature, Vol. 6, 131-164.

JONES, S. y HENSHER, D. (2004), Predicting firm financial distress: A mixed logit model, The Accounting Review, Vol. 79 (4), 1011-1039.

MADDALA, G. (1991), A perspective on the use of limited-dependent and qualitative variables models in accounting research, The Accounting Review, Vol. 66, 788-807.

OHLSON, J. (1980), Financial ratios and the probabilistic prediction of bankruptcy, Journal of Accounting Research, Vol. 18 (1), 109-131.

SANDIN, A y PORPORATO, M. (2007), Corporate bankruptcy prediction models applied to emerging economies. Evidence from Argentina in the years 1991 - 1998, International Jorunal of Commerce and Management, Vol. 17 (4), 295-311.

ZMIJEWSKI, M.E. (1984), Methodological issues related to the estimation of financial distress prediction models, Journal of Accounting Research, Vol. $22(1), 59-82$. 